\title{
An Indoor Variance-Based Localization Technique Utilizing the UWB Estimation of Geometrical Propagation Parameters
}

\author{
Brecht Hanssens, David Plets, Member, IEEE, Emmeric Tanghe, Member, IEEE, \\ Claude Oestges, Fellow, IEEE, Davy P. Gaillot, Member, IEEE, Martine Liénard, Taoyong Li, \\ Heidi Steendam, Senior Member, IEEE, Luc Martens, Member, IEEE, and Wout Joseph, Senior Member, IEEE
}

\begin{abstract}
This work presents a novel localization framework based on Ultra-Wideband (UWB) channel sounding, employing a triangulation method using the geometrical properties of propagation paths, such as time of arrival, angle of departure, angle of arrival, and their estimated variances. In order to extract these parameters from the UWB sounding data, an extension to the high-resolution RiMAX algorithm was developed, facilitating the analysis of these frequency-dependent multipath parameters. This framework was then tested by performing indoor measurements with a vector network analyzer and virtual antenna arrays.

The estimated means and variances of these geometrical parameters were utilized to generate multiple sample sets of input values for our localization framework. Next to that, we consider the existence of multiple possible target locations, which were subsequently clustered using a Kim-Parks algorithm, resulting in a more robust estimation of each target node. Measurements reveal that our newly proposed technique achieves an average accuracy of $0.26 \mathrm{~m}, 0.28 \mathrm{~m}$ and $0.90 \mathrm{~m}$ in Line-of-Sight (LoS), Obstructed-LoS (OLoS), and Non-LoS (NLoS) scenarios, respectively, and this with only one single beacon node. Moreover, utilizing the estimated variances of the multipath parameters proved to enhance the location estimation significantly compared to only utilizing their estimated mean values.
\end{abstract}

Index Terms-Channel Sounding, Channel Modeling, Localization, Location Estimation, Location Tracking, Positioning, KimParks, Multipath Clustering, Ultra-Wideband, RiMAX, Indoor.

\section{INTRODUCTION}

$\mathbf{I}$ $\mathrm{N}$ recent years, there has been a growing interest in the application of wireless sensor networks (WSNs). Location information of sensor nodes in WSNs is crucial for many applications, for example target localization, tracking and guidance of mobile nodes. A possible method to obtain this information is equipping each node with a GPS receiver, which is a rather expensive and energy-consuming approach focusing mostly on outdoor environments only. In most WSNs, only a limited number of all sensor nodes know their exact position; these are called the beacon nodes. The mobile nodes can estimate their position relative to these beacon nodes, and are called the target nodes. Many alternative approaches to GPS can be adopted to estimate the location of these target nodes, most of which are either based on a triangulation method using angle of arrival (AoA) information [1], [2], a

C. Oestges is with ICTEAM, Université catholique de Louvain, Belgium. D. P. Gaillot and M. Liénard are with IEMN, University of Lille 1, France. T. Li and H. Steendam are with TELIN, Ghent University, Belgium.

B. Hanssens, D. Plets, E. Tanghe, L. Martens and W. Joseph are with WAVES, Ghent University - imec, Belgium. Email address of the corresponding author: Brecht.Hanssens@UGent.be trilateration approach using time of arrival (ToA) [3], [4] or received single strength indication (RSSI) [5]. However, these methods mainly focus their efforts on Line-of-Sight (LoS) scenarios and for direct propagation paths only, without taking reflection or transmission into account. RSSI-based methods compare the on-site measured RSSI values to the ones stored in a fingerprinting database. This is a rather cumbersome and often very inaccurate approach, which requires carrying out a-priori measurements or simulations in the environment, and is very geometry- and site-dependent. Cooperative estimation schemes can be found in the literature as well. For example, [6] proposes a localization scheme to estimate the AoA by comparing the measured RSSI values of multiple beacon signals received at two perpendicularly-oriented antennas at the target node. Their experiments demonstrate an average accuracy of $1.24 \mathrm{~m}$, but only consider simplified LoS-scenarios without the presence of scattering objects.

Therefore, we propose a measurement-based ray tracing method exploiting the geometrical properties of propagation paths, to tackle the problem of target localization using UltraWideband (UWB) channel sounding, working accurately in LoS, Obstructed-LoS $(\mathrm{OLoS})$, as well as Non-LoS (NLoS) scenarios. UWB is a popular technology in WSNs, allowing for very high data rates over a short distance due to its large bandwidth. This broad bandwidth also implies a high temporal resolution, making it suitable to achieve a high ranging accuracy, and thus allows for the precise positioning of each target node in the network. UWB communication is characterized by its ability to transmit short pulses with low power spectral density in a large frequency band (ranging from $3.1 \mathrm{GHz}$ to $10.6 \mathrm{GHz}$ ). This allows communication systems to harmlessly operate in frequency bands currently occupied by other applications. In current literature, localization based on UWB [7]-[10] focuses its efforts on the trilateration of the unknown location of a target node using three or more beacon nodes. Moreover, in contrast to our proposed method, these methods only work in LoS situations.

The main advantage of our technique is that it identifies the location of a target node using only one single beacon node, making our solution inherently different from existing ones, which need a minimum of three nodes or more. Next to that, our approach can handle OLoS and NLoS scenarios exceptionally well, due to the fact that we combine the AoA with the angle of departure (AoD) information of each propagation path at the beacon node. This allows us to easily differentiate between direct- and scattered propagation paths, and employ different strategies for the localization of a target node accordingly. As 
such, we can cover the entire indoor environment with fewer nodes in contrast to the more contemporary methods breaking down the environment into LoS-areas, and thus needing more beacon nodes doing so. Localization algorithm using a single beacon node have previously been proposed in [11], [12], but they focus on cooperative localization approaches with the necessity of multiple users (target nodes), whilst our work focuses on a single beacon and a single user (target) in order to localize the latter one.

In our work, we have utilized both the mean- and the variance estimates of the extracted geometrical multipath parameters to generate a distribution from which we can sample several input parameter sets for our localization framework, in contrast to only using their mean values. We also consider the existence of multiple possible estimated target locations, in comparison with the aforementioned works which estimate only one single target location per measurement [1]-[4], [6][10]. This is a rather different, but at the same time a very robust approach with respect to localization. Because the RiMAX [13] multipath estimation framework allows us to extract several propagation paths per measurement, we end up with a mixture of direct paths between transmitter and receiver, and multiple scattered paths. This allows us to cluster all these possible locations (originating from direct- and scattered paths), as was done before in [14]. In contrast to their work, we consider the existence of multiple clusters, to improve the robustness of our localization framework to outliers. Moreover, our approach can easily be extended to also estimate the orientation of the target with the help of an extra beacon node, as proposed in [1]. One of the drawbacks of our approach is that we require synchronization between the mobile node and the beacon node, but this can be resolved by applying twoway ranging, in order to compensates for the phase differences between the oscillators of both target- and beacon nodes.

\section{MEAsurements}

\section{A. Measurement environment}

The indoor measurements were conducted in a laboratory of Ghent University in Belgium, as depicted in Figure 1 . The base surface of the long side of the laboratory was approximately $16 \mathrm{~m}$ long and $5 \mathrm{~m}$ wide (see Figure 1(b), and the small side adjacent to it was approximately $8.5 \mathrm{~m}$ long and $5 \mathrm{~m}$ wide (see Figure $1(\mathrm{c})$. This environment was mostly equipped with metallic cabinets, tables, computers and other hardware equipment. As we can see from the pictures below, this environment can be considered as a very cluttered one. We believe that more realistic environments, which are likely to be less cluttered than the one we measured in, will further enhance the accuracy of the localization framework we will explain in the following sections.

In this laboratory environment, the indoor radio channel of 15 spatially distinct receiver positions (target nodes) was measured with respect to one single transmitter (beacon node). We refer to Figure 6(a) for an outline of their positions. In total, 8 of the 15 positions were considered Line-of-Sight (LoS) scenarios, where there is a direct (free space) path from the transmitter to the receiver. Next to that, 4 of the 15

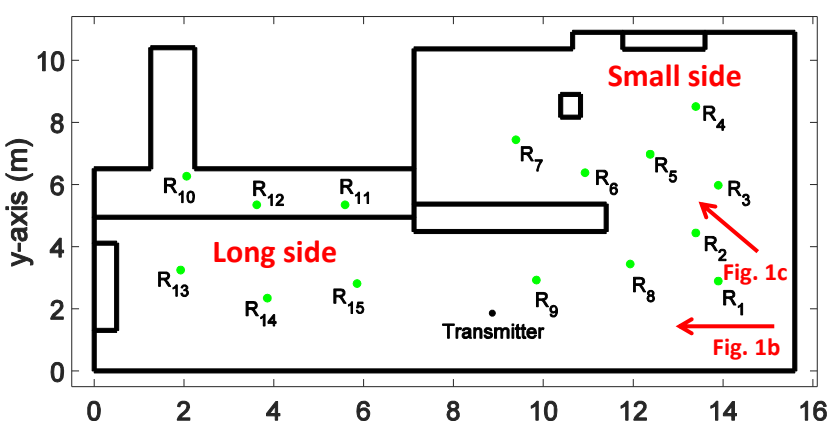

(a) Schematic representation

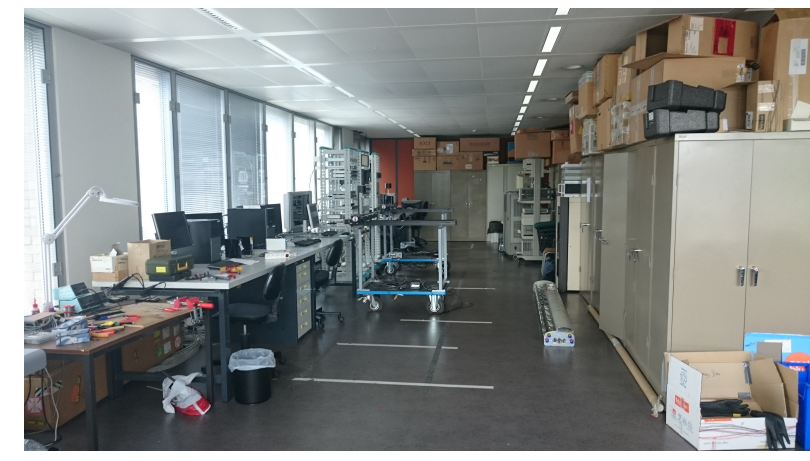

(b) Photograph: long side

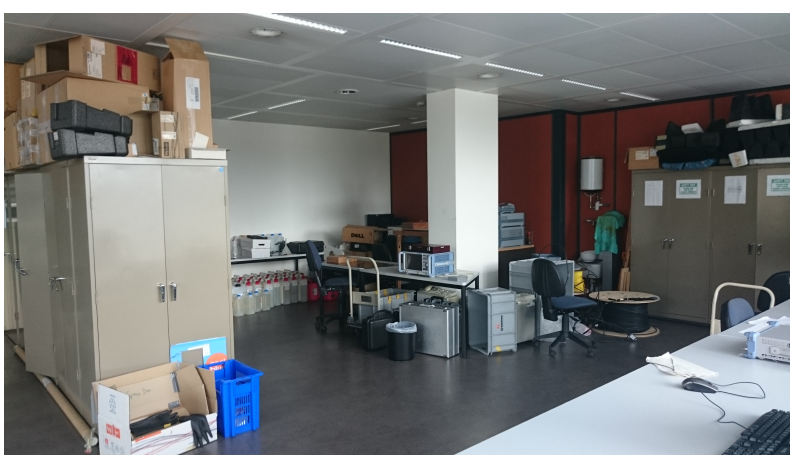

(c) Photograph: small side

Figure 1: Measurement environment.

positions were considered Obstructed Line-of-Sight (OLoS) scenarios, where the free space path from transmitter to the receiver has to undergo a reflection and/or a diffraction. Lastly, 3 of the 15 positions were considered Non Line-of-Sight (NLoS) scenarios, where the (partly free space) path from the transmitter to the receiver needs to undergo a transmission through a certain medium (in this case, a plasterboard wall). The exact positions of the receiver nodes with respect to the transmitter were measured by means of a digital laser distance meter, which had an accuracy of $2 \mathrm{~mm}$.

\section{B. Channel sounding procedure}

Wideband channel sounding measurements were carried out at each of the 15 positions. A Vector Network Analyzer (VNA) of type Rohde \& Schwarz ZNB8 was used to probe the indoor radio channel ranging from $3.1 \mathrm{GHz}$ up to $10.6 \mathrm{GHz}$, this being the UWB frequency band. Both at the transmit and receiveside of the measurement system, a virtual antenna array was 
created by an automated positioning system on which the antennas were mounted. A virtual antenna array offers the benefit that the antennas do not suffer from mutual coupling, which would otherwise distort the radio channel measurements. The VNA was then used to measure the complex gain between each combination of transmit and receive antenna. In the aforementioned UWB band, $M_{f}=7501$ uniformly spaced frequency points were sampled with a resolution bandwidth of $10 \mathrm{kHz}$, resulting in a maximum measurable delay of $1000 \mathrm{~ns}$ with a resolution of $0.13 \mathrm{~ns}$. The feeder cables for the transmit and the receive antenna were included in the VNA calibration in order to exclude them from the measurement data.

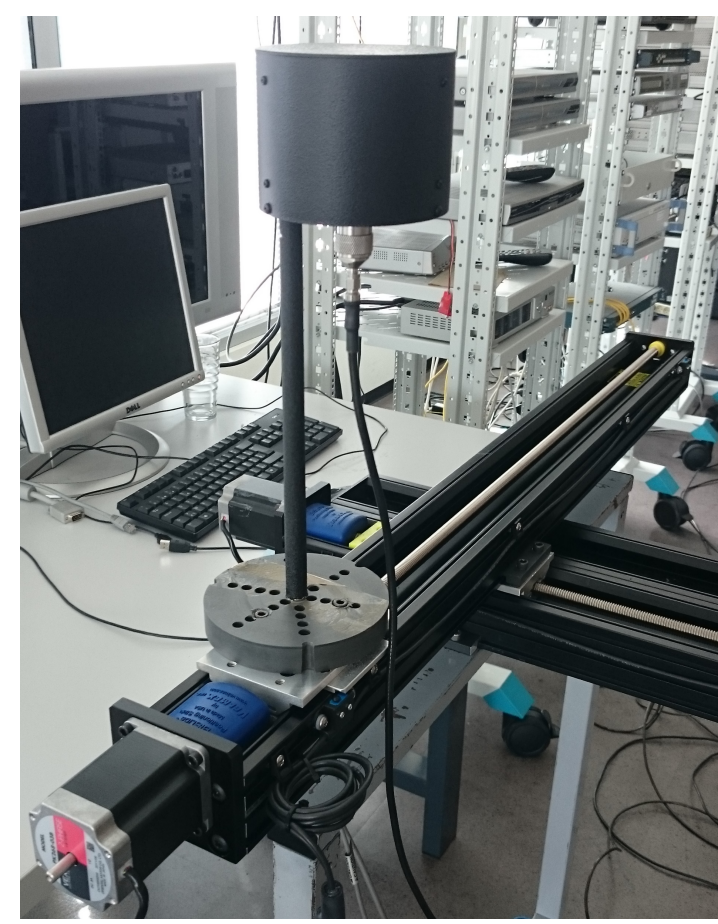

Figure 2: Automated positioning system and UWB antenna, both together forming a virtual Uniform Circular Array (UCA).

At both the transmitting $(\mathrm{T})$ and the receiving $(\mathrm{R})$ side of the measurement system, the virtual array was a planar horizontal Uniform Circular Array (UCA) (see Figure 2). Three different types of configurations for the antenna array were tested, namely a UCA with $\left[M_{T} \times M_{R}\right]=[4 \times 4]$ antennas at transmitter and receiver, a $[6 \times 6] \mathrm{UCA}$, and an $[8 \times 8] \mathrm{UCA}$. The inter-element spacing for this array was 0.45 times the wavelength at the highest measured frequency $(10.6 \mathrm{GHz})$, resulting in a spacing of $1.27 \mathrm{~cm}$ between two adjacent antennas on the UCA circle. The transmitting and receiving antennas were omnidirectional UWB antennas in the azimuth plane of type Electro-Metrics EM-6865 [15], both placed $1.5 \mathrm{~m}$ above ground level. All measurements were done outside of regular working hours, since frequency-swept measurements with virtual arrays require the radio channel to be static, without any form of movement. It should be noted that a real antenna array and advanced channel sounder equipment (e.g., the MIMOSA sounder [16] or the RUSK sounder [17]) can measure this channel in less than a millisecond. By doing so, the channel can be considered static during this period of time, and the requirement that the channel should not contain any movement can be omitted.

\section{EVALUATION}

The flow graph in Figure 3 summarizes the processing steps in our evaluation scheme, and describes how we convert the measurement data into an estimate of the target node location.

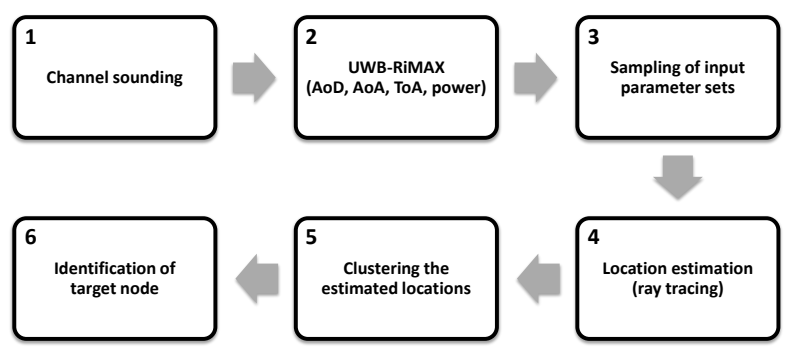

Figure 3: Flowchart of the evaluation process.

The novelty and added value of this work over existing localization techniques can especially be found in bocks 3, 5 and 6 in Figure 3, which will be explained in detail in the following subsections. It should be noted that comparable versions of both (UWB-)RiMAX [13] and the ray tracing algorithm [18] can be found in the literature.

\section{A. UWB-RiMAX algorithm (Figure 3 block 2)}

Our virtual array system with multiple antennas at transmitter and receiver allows us to obtain a Multiple-Input Multiple-Output (MIMO) measurement system, from which we can extract the geometrical properties of the electromagnetic waves, such as the AoD, AoA and ToA. An observation of the frequency response of a MIMO radio channel $\boldsymbol{h}$ can be modeled as the superposition of a deterministic part $s$ (specular multipath components (SMC)) and a stochastic part $\boldsymbol{d}$ (diffuse scattering; dense multipath components (DMC), and additive measurement noise).

$$
\begin{aligned}
& \boldsymbol{h}=\boldsymbol{s}\left(\boldsymbol{\theta}_{\text {smc }}\right)+\boldsymbol{d}\left(\boldsymbol{\theta}_{\text {dan }}\right) \\
& \boldsymbol{h} \in \mathbb{C}^{M_{T} \times M_{R} \times M_{f}} .
\end{aligned}
$$

In this formula, $\boldsymbol{h}$ can be considered as a random variable distributed according to a complex multivariate Gaussian distribution $\boldsymbol{h} \sim \mathcal{N}_{c}\left(\boldsymbol{s}\left(\boldsymbol{\theta}_{s m c}\right), \boldsymbol{R}\right)$. The deterministic part $\boldsymbol{s}\left(\boldsymbol{\theta}_{s m c}\right)$ of the data model acts as the first-order statistics of the radio channel, while the stochastic part $\boldsymbol{d}\left(\boldsymbol{\theta}_{\text {dan }}\right)$ (DMC and noise) describes the second-order statistics by means of the covariance matrix $\boldsymbol{R}$. Both operators $s$ and $\boldsymbol{d}$ reconstruct the deterministic- and stochastic part of the radio channel, and are explained in [13]. Based on the capability of the RiMAX estimation framework to extract both parameter sets from the virtual array measurement data, the following structures for the deterministic and stochastic arrays can be adopted:

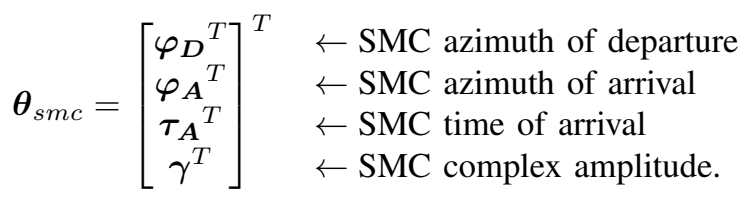


In (2), $\varphi_{D}, \varphi_{A}, \tau_{A}$ are $\gamma$ are $P \times S$ matrices, where $P$ is the number of SMCs extracted from the measurement data, and $S$ is the number of sub-bands in which the total UWB bandwidth was partitioned (in analogy with the UWB-SAGE algorithm, which will be explained in the next section). Each row in these matrices contains the corresponding specular parameter for each of the $p \in \mathcal{P}$ propagation paths $(P=|\mathcal{P}|$ in total), and describes its frequency-dependency in each of the $s \in \mathcal{S}$ sub-bands $(S=|\mathcal{S}|$ in total). Note that the angular modeling was limited to that of the azimuthal plane, and we only consider a single snapshot of the channel. Whilst it would be possible to use several snapshots of the channel in a real measurement environment, we would then have to impose a parametric model to handle the time dependence of the SMC, or assume them to be independent and identically distributed (i.i.d.) across snapshots in time. Since this is out of the scope for the purpose of this paper, we will use a single snapshot of the channel and leave this up for future work.

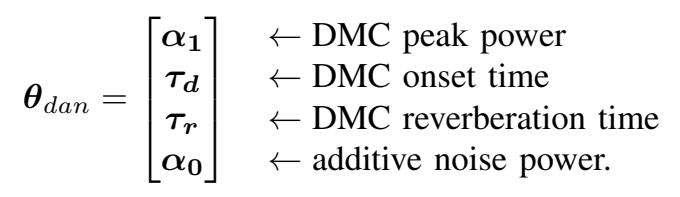

In (3), $\boldsymbol{\theta}_{\text {dan }}$ is a $4 \times S$ matrix containing the DMC and noise (DAN) parameters for each sub-band $s \in S$. The RiMAX data model assumes that the DMC are spatially white at the transmit and receive side of our measurement system, meaning that they have constant angular power densities. It should be noted that recent works (e.g., [19] and [20]) will assume the DMC to be spatially correlated with the SMC, but we leave the influence of this on the localization accuracy for future work. Furthermore, the DMC is assumed correlated and wide-sense stationary in the frequency domain. The DMC and noise power describes how the power of the diffuse signal is distributed over the time-delay domain. The DMC and noise power is commonly modeled based on the observation that its Power Delay Profile (PDP) $\psi(\tau)$ has a base delay $\tau_{d}$ related to the distance between the transmitter and receiver, together with an exponential decay over time-delay (see Eq. (4)), corrupted by complex additive white Gaussian noise with power $\alpha_{0}$. The parameter $\alpha_{0}$ thus describes the noise floor, whilst $\alpha_{1}+\alpha_{0}$ describes the peak power at the time-delay $\tau_{d}$, from which the PDP follows an exponential decay regulated by $\tau_{r}$ (reverberation time). The reverberation time is the time for the electromagnetic waves to uniformly distribute themselves in a room. Higher values for $\tau_{r}$ indicate a slow exponential decay of the PDP, whilst low values indicate a fast decay. A full discussion of this model can be found in [13].

$$
\psi(\tau)=\alpha_{1} \exp \left(-\frac{\tau-\tau_{d}}{\tau_{r}}\right)+\alpha_{0} \quad\left(\tau>\tau_{d}\right) .
$$

The idea of the localization framework is to only work with the reliable deterministic contributions of the SMC, since the stochastic contributions of the DMC (comprised of diffuse scattered paths and weak SMC) are unreliable to perform the ray-matching of the propagation paths, which is the basis for our localization framework on. Hence, we need the distinction between SMC and DMC.
We note that the (UWB-)RiMAX framework is an iterative maximum-likelihood (ML) algorithm; the estimated ML parameter sets $\hat{\boldsymbol{\theta}}_{s m c}$ and $\hat{\boldsymbol{\theta}}_{d a n}$ (denoted with a hat-operator) of the 'true' values of $\boldsymbol{\theta}_{s m c}$ and $\boldsymbol{\theta}_{d a n}$ are estimated such that they maximize the likelihood of observing the measured frequency response $\boldsymbol{h}$ of the radio channel given these parameters.

The original data model of the RiMAX estimation framework follows the narrowband assumption, hence stating that the SMC and DMC are Kronecker-separable in the spatial and frequency domains in order to keep the algorithm computationally feasible [13]. As such, it does not account for frequencydependent propagation phenomena, so that the reflection coefficients of certain materials in the environment (e.g., metallic cabinets) can no longer be considered constant with respect to frequency. In addition, the antenna array responses of transmitter and receiver will no longer be frequency-independent, and can vary significantly over the total UWB bandwidth. In order to track these frequency-dependent propagation parameters, an extension to the RiMAX framework was developed that can process UWB measurement data, and will be referred to as UWB-RiMAX from now on [21]. This framework is described in Algorithm 1, and depicted in Figure 4. Prior to the processing of the measurement data, the entire UWB band was split up into $S=30$ sub-bands of $250 \mathrm{MHz}$ to assure frequency stationarity (and thus Kronecker separability) in each sub-band, following the multi-band UWB principle.

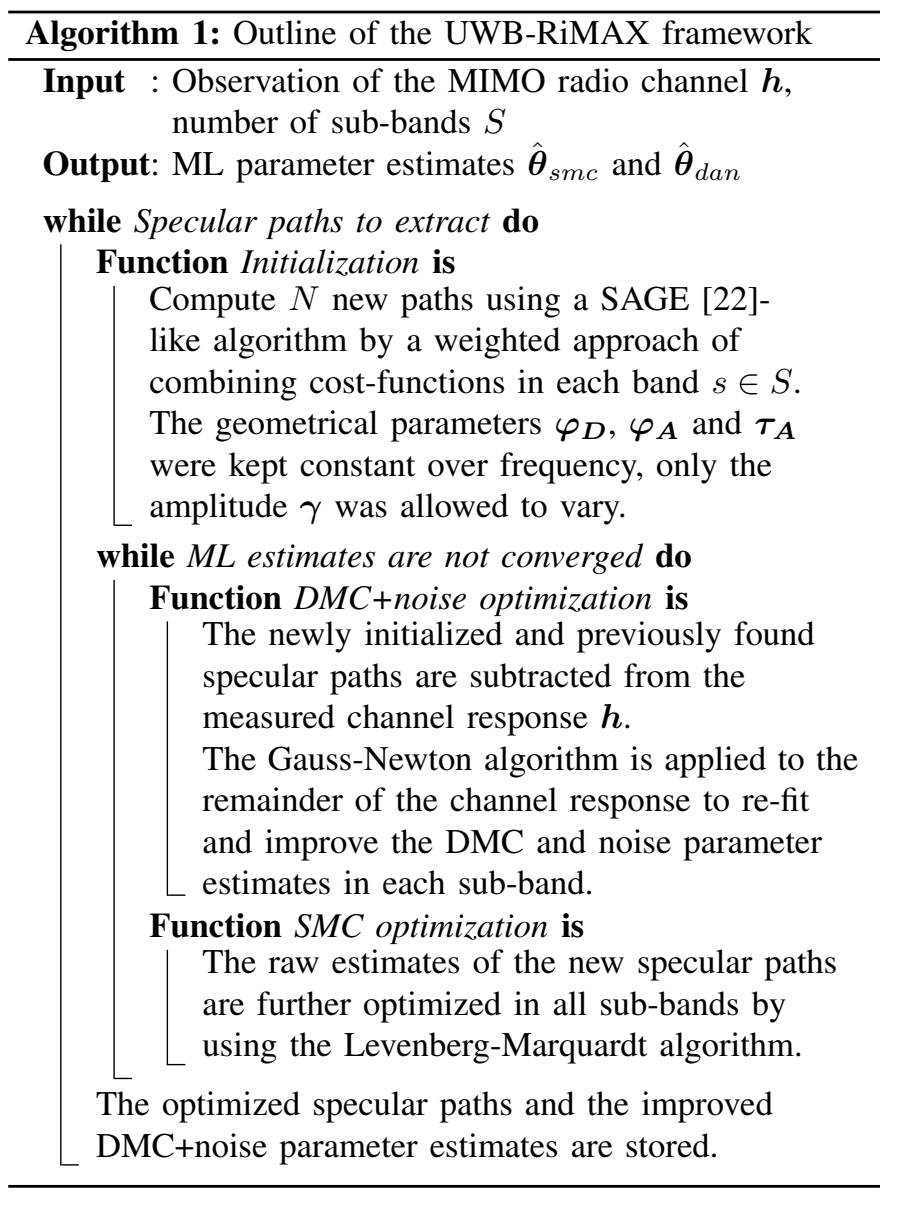

The algorithm keeps searching for $N$ new specular paths per 


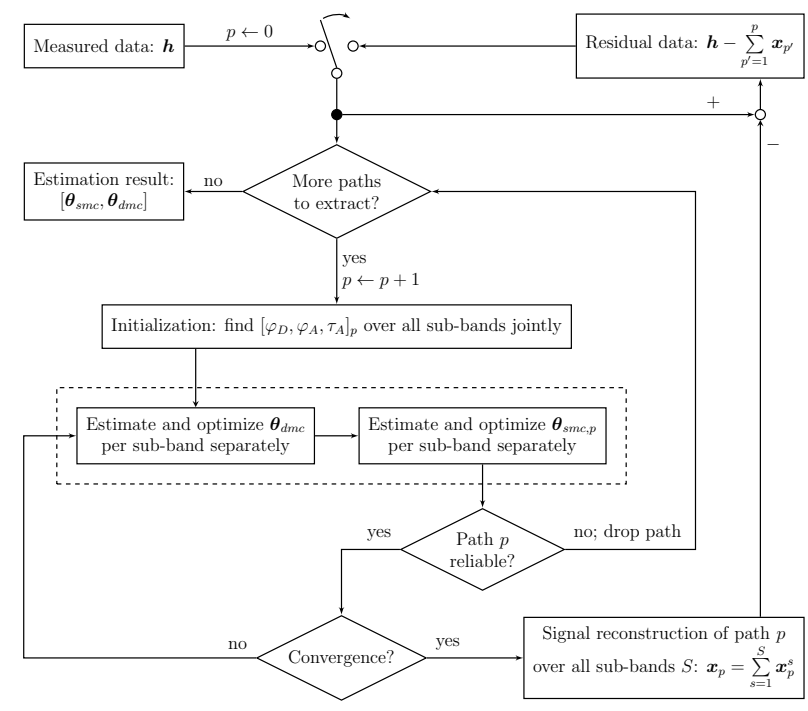

Figure 4: Flowchart of the UWB-RiMAX multipath estimation algorithm. In the first iteration, the input data is the measured channel $\boldsymbol{h}$. The switch at the top will ensure that subsequent iterations of the algorithm use the residual channel as its input data.

iteration (so that their mutual dependency can be taken into account) until the stopping criterion that is described in [13] is met. When this stopping criterion is met, we will have a total of $P$ propagation paths. The approach outlined in this work is based on the estimated power of the extracted propagation paths. For each path, we associate a Signal-to-Noise Ratio (SNR) with it, based on its estimate for the complex amplitude, and its estimation error according to the Fisher information matrix (described in Section III-B). A propagation path is considered to be unreliable and hence removed from further analysis if its estimated SNR is smaller than the $90^{\text {th }}$ percentile of a chi-squared distribution with a single degree of freedom, equal to $4.32 \mathrm{~dB}$ [23].

One important aspect to note is that the (UWB-)RiMAX algorithm was implemented in such a way that it is optimized to execute in real-time. For example, in [17] RiMAX was used to measure mobile channels. The computation time needed to perform the variance-based localization detailed below, is only a fraction of the time needed to execute the RiMAX algorithm.

\section{B. Variance-based localization (Figure 3, block 3)}

After the estimation of the geometrical multipath parameters over all sub-bands in the previous subsection, we will take the median of each geometrical parameter $\left(\varphi_{D}, \varphi_{A}\right.$, and $\left.\tau_{A}\right)$ over all sub-bands in order to keep the localization algorithm computationally viable. This ensures that we get the mostlikely specular propagation paths in the environment over all sub-bands. This can be mathematically formulated as follows:

$$
\begin{aligned}
\tilde{\varphi}_{D} & =\operatorname{atan}_{2}\left(\operatorname{median}\left\{\sin \varphi_{\boldsymbol{D}}\right\}, \operatorname{median}\left\{\cos \varphi_{\boldsymbol{D}}\right\}\right) \\
\tilde{\varphi}_{A} & =\operatorname{atan}_{2}\left(\operatorname{median}\left\{\sin \varphi_{\boldsymbol{A}}\right\}, \operatorname{median}\left\{\cos \boldsymbol{\varphi}_{\boldsymbol{A}}\right\}\right) \\
\tilde{\tau}_{A} & =\operatorname{median}\left\{\boldsymbol{\tau}_{\boldsymbol{A}}\right\}
\end{aligned}
$$

In $\mathrm{Eq}(5)$ and $\mathrm{Eq}(6)$, the $\operatorname{atan}_{2}$ operator was used to ensure that we get the circular median of the AoD and AoA parameters. Processing the results in each sub-band separately would result in the fact that we would have to perform the localization $S=30$ times with the input parameters per sub-band.

A significant feature of the RiMAX algorithm is that it also provides an estimate of the Fisher information matrix (FIM) in its output. For asymptotically uncoupled parameters, the diagonal elements of the inverse of this FIM are the estimated variances of the propagation parameters in Eq. (2) and Eq. (3). In our localization framework, we will combine both the estimated mean values of the geometrical parameters with their corresponding estimated variances, prior to the estimation of the location of the receiver. More specifically, we start by selecting a number of propagation paths per measurement position, accounting for a certain percentage of the total power in the channel. In LoS scenarios, few paths will account for most of the total power, but more paths are needed in OLoS or NLoS scenarios to account for the same percentage of the total power. This inherently adapts our framework to account for the difference between LoS and OLoS or NLoS scenarios. This was done by summing the powers $\gamma$ of each propagation path over all sub-bands $S$, and afterwards sorting them in descending order. After this, we will associate a normal distribution with each propagation path parameter (AoD, AoA and ToA), based on its estimated mean value and its corresponding estimated variance. We will then sample different sets of input values for our ray tracing algorithm from these distributions, with a weighting based on the estimated power of each propagation path, until we have a set of so-called 'virtual paths' (arbitrarily chosen as 10 times the original number of paths accounting for a certain percentage of the total power in the channel). Stronger estimated paths will thus be sampled more often than weaker paths, although the latter ones might still provide us with useful information for our localization framework.

The UWB-RiMAX algorithm is constrained so that it estimates a maximum of 50 propagation paths. However, we have found that due to the implemented stopping criterion, the algorithm stops searching for new paths before this maximum number is reached. Table $[$ summarizes the number of strongest propagation paths accounting for a certain percentage of the total measured power in the channel:

Table I: Number of propagation paths accounting for a certain percentage of the measured power.

\begin{tabular}{|c|c|c|c|c|}
\hline \multicolumn{2}{|c|}{$\begin{array}{c}\text { Nr. of paths accounting for } \\
x \% \text { of the measured power }\end{array}$} & $\begin{array}{c}\text { LoS } \\
\text { scenario }\end{array}$ & $\begin{array}{c}\text { OLoS } \\
\text { scenario }\end{array}$ & $\begin{array}{c}\text { NLoS } \\
\text { scenario }\end{array}$ \\
\hline \multirow{3}{*}{$4 \times 4$ array } & $90 \%$ & 7 & 6 & 10 \\
& $95 \%$ & 9 & 8 & 14 \\
\hline \multirow{4}{*}{$6 \times 6$ array } & $99 \%$ & 15 & 13 & 21 \\
& $90 \%$ & 8 & 12 & 23 \\
& $99 \%$ & 11 & 20 & 30 \\
$8 \times 8$ array & $90 \%$ & 17 & 31 & 40 \\
& $95 \%$ & 13 & 14 & 21 \\
& $99 \%$ & 20 & 35 & 22 \\
\hline \multirow{3}{*}{8} & 99 & & & 40 \\
\hline
\end{tabular}

From Table I] we can state that by working with $90 \%$ of the total power in the channel measured with an $8 \times 8$ antenna-array, this corresponds with 9 paths on average in a LoS scenario, 14 paths in an OLoS scenario and 21 paths in an 
NLoS scenario. We can also observe that a larger antenna-array configuration is able to estimate more paths (distinguish more paths) from the measurement data for the same percentage of total power in the channel. Our localization framework will thus sample a total of 90 virtual paths on average in a LoS scenario, 140 virtual paths in an OLoS scenario and 210 virtual paths in an NLoS scenario. Since the UWB-RiMAX algorithm estimates 5 new propagation paths per iteration with a maximum of 50 paths, it estimates more than $99 \%$ of the total power in the channel within 10 iterations.

We found that the measured path loss varied between $-26.2 \mathrm{~dB}$ and $-19.4 \mathrm{~dB}$ for the various $\mathrm{LoS}$ scenarios, between $-30.2 \mathrm{~dB}$ and $-28.4 \mathrm{~dB}$ for the various OLoS scenarios and between $-38.5 \mathrm{~dB}$ and $-35.2 \mathrm{~dB}$ for the various NLoS scenarios. In future work, this information could be useful to distinguish between estimating the receiver in the same room, or in an adjacent room. This distinction can also be performed by looking at the number of estimated propagation paths accounting for a certain percentage of the power. In an OLoS scenario, this was on average more than one and a half the number of paths in a LoS scenario, and in an OLoS scenario, this was on average more than double.

\section{Measurement-based ray tracing (Figure 3, block 4)}

In this section, we will explain how the known location of the beacon node transmitter $\left[\mathrm{T}_{\mathrm{x}}, \mathrm{T}_{\mathrm{y}}\right]$, together with the geometrical propagation parameters $\varphi_{D}, \varphi_{A}$, and $\tau_{A}$ of our virtual parameter set can be used to estimate the unknown location of a target node receiver $\left[\mathrm{R}_{\mathrm{x}}, \mathrm{R}_{\mathrm{y}}\right]$. The ray tracing framework we will use for this purpose is comparable to the one described in [18]. In their work, a measurementbased ray tracer for multi-link double directional propagation parameters was developed to identify the scattering points of the propagation paths, by tracing these estimated rays from a known transmitter location to a known receiver location. In contrast to their work, we will use such a framework to estimate the unknown receiver location by using an a-priori known map of the environment in combination with tracing the virtually constructed rays (paths) originating from a known transmitter location.

In our work, we thus assume that we have access to a map of the environment of the surrounding area near the transmitter. This can be a very simple map describing only the walls (possibly supplemented with the materials they were made of), but it can be extended with information about where certain larger objects are located (e.g., metallic cabinets). The information contained in this map will be used to construct the trajectory of the propagation paths from the known transmitter's location into the environment. Since we have estimates of the angular information of each path (AoD and AoA) and its associated path length (based on the ToA information), we can estimate how each path physically propagated from the transmitter to where the travel time along its trajectory equaled the ToA (since both are related by the speed of light). This final position is then regarded as an estimated location of the receiver. Algorithm 2 describes this measurement-based ray tracing localization algorithm.

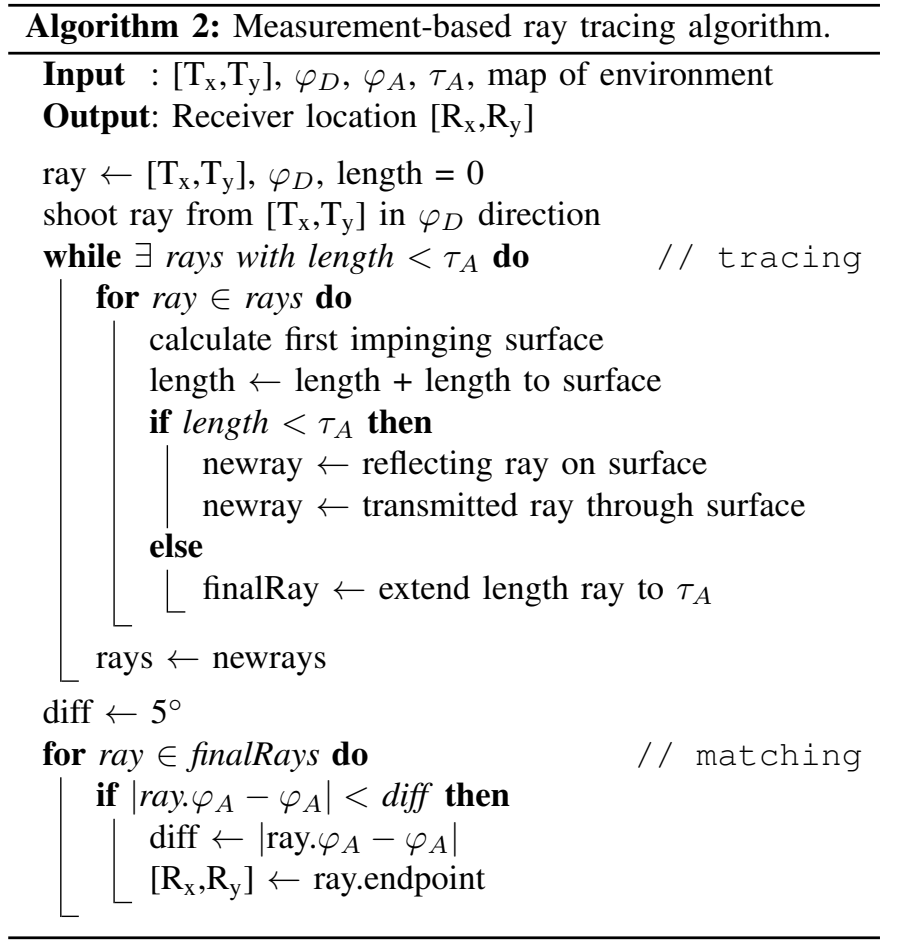

In this algorithm, the first phase performs the actual ray tracing. That is, launching a ray, calculating where it impinges on a surface, and performing a reflecting- and a transmission operation. The second phase performs the ray matching, in which the ray with the tracked AoA at the final location which best matches the UWB-RiMAX estimated AoA is considered the most-likely propagation path associated with the input set of geometrical propagation parameters. Of all virtual paths, not all will satisfy this ray matching selection criterion (thus having a sufficiently small difference between the ray traced AoA and the estimated AoA). Our algorithm keeps sampling new input sets until we achieve the predefined number of virtual paths for each measurement positions (which can vary over a LoS, OLoS or NLoS scenario). It should be noted that we consider up to 3 reflections and/or transmissions of the rays in the environment, to keep the algorithm viable and not accumulate errors due to the limitation of the accuracy with which the map of the environment was implemented.

The accuracy with which this map was implemented will have an influence on the estimation accuracy of the target node for OLoS and NLoS scenarios where the signal impinges at the receiver following a reflection and/or transmission in the environment. For LoS scenarios, the direct path will have a greater influence in the location estimation algorithm than the reflected paths, such that this scenario suffers less from the accuracy with which the map of the environment was implemented. Even so, we believe that if an error of $10 \mathrm{~cm}$ was made when entering the dimensions of the environment, this would at most result in an additional error of $20 \mathrm{~cm}$ $(2 \times 10 \mathrm{~cm})$ for the location estimate of the target node. 


\section{Clustering of estimated locations (Figure 3 block 5)}

In our work, we have a multitude of (virtual) propagation paths in our parameter set, giving rise to multiple estimated target locations. This assumption is in line with reports in the literature which found that propagation paths arrive at the receiver in clusters (e.g., COST 259 [24] and COST 273 model [25]). This is a rather different approach with respect to localization, but it proves to be a very robust method when we look at the ultimate estimation of the target node.

Since our UWB-RiMAX multipath estimation framework allows us to extract several propagation paths per measurement position, which are then used to sample new virtual paths from, we can end up with a mixture of direct- and scattered paths between transmitter and receiver. Some of these paths can be false due to errors in the estimation framework, and some of the ray traced estimated locations can be incorrect due to the finite resolution of our ray tracing algorithm (more specifically, the accuracy of the modeled environment). This leads to the existence of multiple target location estimates, which can be a mixture of valid and invalid estimates. Therefore, we aim to cluster these estimated target locations. In contrast to the work in [14], we consider the existence of multiple clusters, to improve the robustness of our localization scheme to outliers. By doing so, we try to group the valid estimates for the target node location, and disregard the erroneous ones. Afterwards, we need to make an ultimate estimation for the target node location, by formulating a suitable selection criterion.

We first define a clustering-metric to find the optimal number of clusters, in which to partition the total set of estimated receiver locations. The Kim-Parks clustering index [26] was used for this purpose, which employs two partition functions showing opposite properties around the optimal cluster number. We can define the under-partition measure function $v_{u}(k, \mathbf{V} ; \mathbf{Z})$ of the Kim-Parks index as follows:

$$
v_{u}(k, \mathbf{V} ; \mathbf{Z})=\frac{1}{k} \sum_{i=1}^{k} \sum_{\mathbf{z} \in \mathcal{Z}_{i}} \frac{\left\|\mathbf{v}_{i}-\mathbf{z}\right\|}{m_{i}}, \quad 2 \leq k \leq k_{\max }
$$

In this function, $\mathbf{Z}=\left[\mathbf{z}_{1}^{\mathrm{T}}, \mathbf{z}_{2}^{\mathrm{T}}, \ldots \mathbf{z}_{N}^{\mathrm{T}}\right]^{\mathrm{T}}$ is a matrix of $N$ receiver estimates, where $\mathbf{z}_{n}$ is a $1 \times 2$ vector representing a set of (x,y)-coordinates for the estimated location of the $n^{\text {th }}$ receiver index. $\mathbf{V}=\left[\mathbf{v}_{1}^{\mathrm{T}}, \mathbf{v}_{2}^{\mathrm{T}}, \ldots \mathbf{v}_{k}^{\mathrm{T}}\right]^{\mathrm{T}}$ is a $k \times 2$ matrix of $k$ estimated clusters, where $\mathbf{v}_{i}$ is a two element vector representing a set of (x,y)-coordinates for the estimated location of the $i^{t h}$ cluster. Next to that, $\mathcal{Z}_{i}$ is the set of estimated receiver locations belonging to the $i^{t h}$ cluster, and $m_{i}=\left|\mathcal{Z}_{i}\right|$ is the number of locations in the $i^{\text {th }}$ cluster. This under-partition measure function $v_{u}(k, \mathbf{V} ; \mathbf{Z})$ also represents the mean intra-cluster distance, averaged over all possible clusters $k$. In our work, we used $k_{\max }=7$, which proved to be a feasible maximum number of possible clusters.

The over-partition measure function $v_{o}(k, \mathbf{V})$ of the KimParks index can be defined as follows:

$$
v_{o}(k, \mathbf{V})=\frac{k}{d_{\min }}=\frac{k}{\min _{i \neq j}\left\|\mathbf{v}_{i}-\mathbf{v}_{j}\right\|}, \quad 2 \leq k \leq k_{\max },
$$

in which the denominator $d_{\min }$ is the minimum distance between cluster centers, measuring inter-cluster separation.
The Kim-Parks index can then be formulated as the summation of the normalized versions of these under- and overpartition functions, after which the optimal cluster number $k_{o p t}$ is simply the smallest value of this index for $2 \leq k \leq k_{\max }$.

\section{E. Identification of target node location (Figure 3, block 6)}

After clustering the total set of estimated receiver locations, we have to decide which cluster is most likely to contain the true location of the target node. Therefore, we first prune the clusters by deleting those which have less than a third of the elements in the largest cluster (i.e., the cluster with the most estimated locations in it). Next to that, we also prune the estimated receiver locations in each cluster $i$ by removing those locations $\mathbf{z} \in \mathcal{Z}_{i}$ which lie further away than an arbitrarily chosen 1.5 times the intra-cluster distance $\mathrm{ICD}_{i}$ (see Eq. (10p) from their cluster centroid position $\mathbf{v}_{i}$, in order to improve robustness.

$$
\operatorname{ICD}_{i}\left(k_{o p t}, \mathbf{V} ; \mathbf{Z}\right)=\sum_{\mathbf{z} \in \mathcal{Z}_{i}} \frac{\left\|\mathbf{v}_{i}-\mathbf{z}\right\|}{m_{i}}, \quad 1 \leq i \leq k_{o p t}
$$

In Eq. 10, $\operatorname{ICD}_{i}$ represents the average distance in the cluster $i$ between the estimated receiver locations in that cluster, and the cluster centroid position $\mathbf{v}_{i}$. Clusters with lower ICD thus represent groups of estimated receiver positions of which their locations lie close to each other. Pruning those receiver locations further away than 1.5 times the ICD results in denser and more compact clusters, where their centroid positions are influenced less by their location outliers.

Ultimately, we still have to select the cluster that is most likely to contain the true location of the target node. Therefore, we have established a new decision criterion as follows, taking into account the number of locations $m_{i}$ (= propagation paths) in each cluster $i$, the number of reflections and/or transmissions $\mathbf{r}_{\mathbf{z}}$ each propagation path $\mathbf{z} \in \mathcal{Z}_{i}$ underwent (thus $0,1,2$ or 3 at most), and their distance to the cluster centroid position $\mathbf{v}_{i}$ :

$$
\mathrm{C}_{i}\left(k_{\text {opt }}, \mathbf{V} ; \mathbf{Z}\right)=\frac{\sum_{\mathbf{z} \in \mathcal{Z}_{i}}\left(2^{\mathbf{r}_{\mathbf{z}}} \cdot\left\|\mathbf{v}_{i}-\mathbf{z}\right\|\right)}{\sum_{\mathbf{z} \in \mathcal{Z}_{i}} 2^{\mathbf{r}_{\mathbf{z}}}} \cdot\left(1-\frac{m_{i}}{N}\right)
$$

Adding the parameter $\mathbf{r}_{\mathbf{z}}$ adds more weight in Eq. (11) to those clusters having propagation paths with multiple reflections and/or transmissions. This allows us to prioritize those clusters with fewer interactions with the environment (thus less accumulated errors in the estimation of the receiver location), as we can see that the above criterion becomes lower for clusters with low intra-cluster distance, a low number of reflections and/or transmissions per path, and many paths $m_{i}$ in that cluster. The optimal cluster $i_{\text {opt }}$ is then the one with the lowest criterion $\mathrm{C}_{i}$ with at least two paths in it (otherwise $\left\|\mathbf{v}_{i}-\mathbf{z}\right\|$ would be zero, and thus the criterion itself would be zero), after which the target node location is simply its centroid position $\mathbf{v}_{i}$.

In our evaluation of the localization results, we will compare two closely related algorithms. The first one is 'method 1' [27], which follows the procedure described above, but only uses the estimated mean parameters of the propagation paths for the ray tracing localization. More specifically, the flowchart for this method can be obtained by omitting block 3 from 
Figure 3 (described in Section III-B). The second one is 'method 2', which is the full procedure described above, and thus extends the number of input propagation paths for the ray tracing localization by sampling new virtual paths utilizing the estimated means and the estimated variances of the extracted propagation paths.

\section{RESUlts}

\section{A. Location estimation}

Figure 5 shows an example of the measurement-based ray tracing, clustering and target node localization for the $4^{\text {th }}$ measurement position (see Section II-A). Figures 5(a) and 5(b) depict the results using method 1 (ray-tracing with estimated paths), Figures 5(c) and 5(d) depict the results using method 2 (ray-tracing with virtual paths based on sampling).

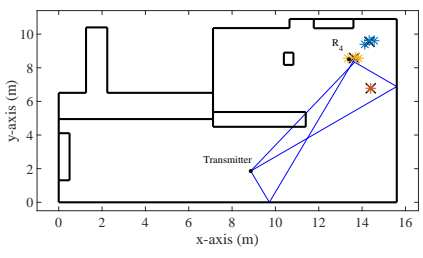

(a) method 1 - without pruning

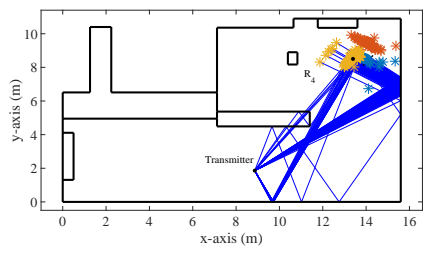

(c) method 2 - without pruning

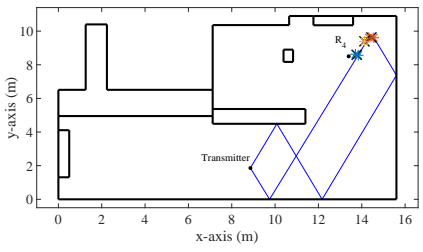

(b) method 1 - with pruning

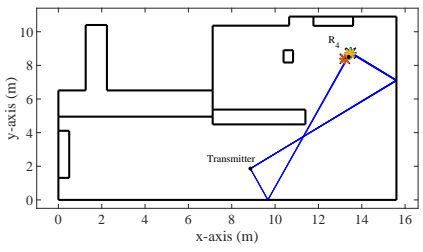

(d) method 2 - with pruning
Figure 5: Measurement-based ray tracing, clustering and localization of the $4^{\text {th }}$ measurement position. Possible receiver positions are indicated with an asterisk, colors indicate their respective clusters, of which the centers are indicated with a black cross. Propagation paths were drawn in blue for the optimal cluster.

From Figure 5, we can see that the cluster pruning operation removes smaller clusters, of which if they were to be chosen as the optimal cluster, they would result in an inaccurate location estimation of the target receiver node. After this pruning, the remaining location estimations are clustered again, as we can clearly see in the transition from Figure 5(c) to Figure 5(d) Looking at this transition, we can also see the robustness of the algorithm, as more and more inaccurate clusters and receiver locations are removed from our dataset.

Figure 6 presents a map of the measurement environment with the location of the transmitter, as well as the true- and estimated receiver locations. From this figure, we can state that there were no major errors in the localization of the receivers, especially for larger antenna array sizes, and that the most inaccurate estimates occurred for the NLoS scenario. As expected, it is very difficult to achieve a good accuracy in an NLoS scenario due to the electromagnetic waves having to propagate through a specific medium in a realistic environment (in this case it was through plasterboard walls).

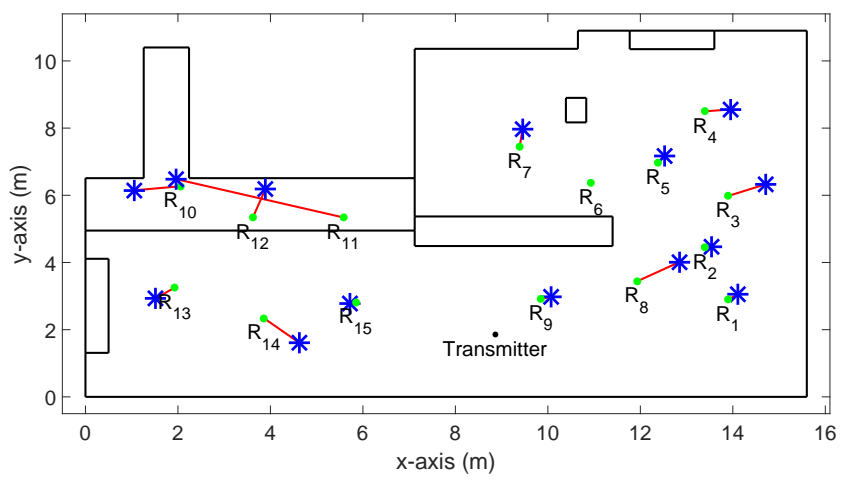

(a) Localization using a $4 \times 4$ array.

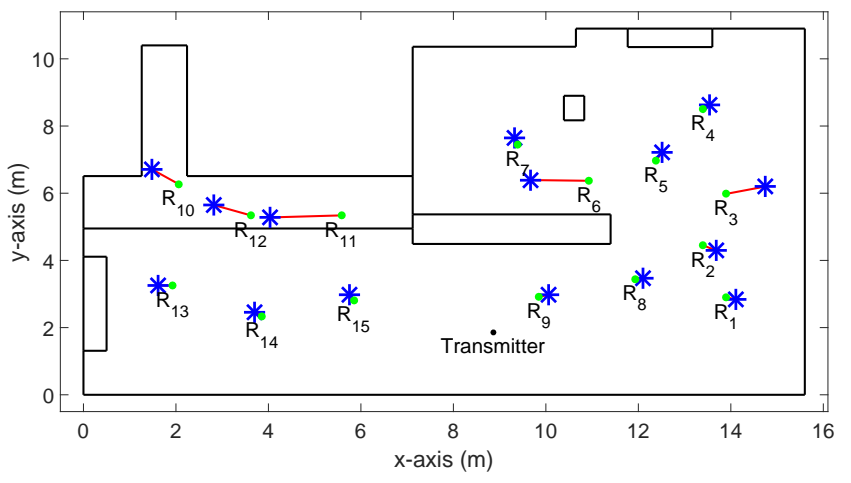

(b) Localization using a $6 \times 6$ array.

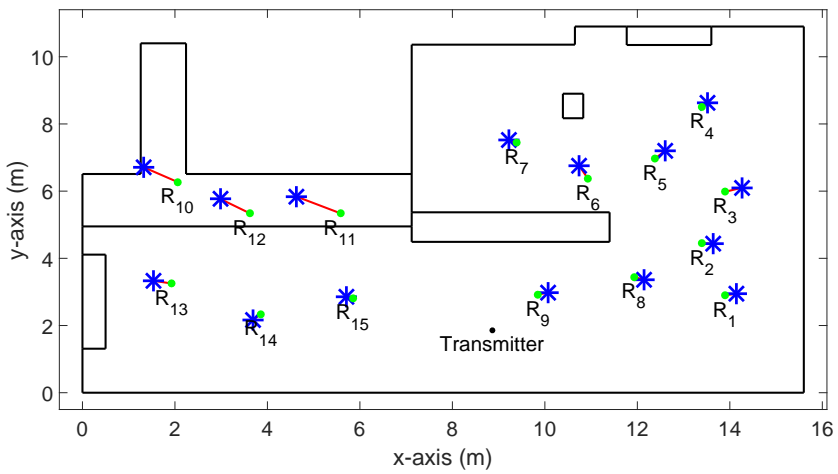

(c) Localization using a $8 \times 8$ array.

Figure 6: Location estimation of the measurement campaign. The true receiver positions are indicated with a green dot, their estimated positions are indicated with a blue asterisk, and both are connected to each other by means of a red line, which thus represents the estimation error for each position.

\section{B. Overview of localization results}

From Table II we can state that our new proposed localization algorithm (method 2) outperforms method 1 most of the time. For example, when we look at the results of the $8 \times 8$ array, we can see that our new technique results in a better average localization of the target receiver nodes for $\mathrm{LoS}$, OLoS, and NLoS scenarios compared to method 1 (given that method 1 fails to estimate the 11th receiver index). Next to that, method 1 was unable to estimate receiver index 11 with a $4 \times 4$ - or an $6 \times 6$ array. This implies that incorporating the variance of the estimated geometrical propagation paths in our localization algorithm contributes significantly to the accuracy, compared to only using the estimated mean values 
Table II: Localization accuracy (in meters) for LoS, OLoS, and NLoS scenarios for different receiver positions, as a function of antenna array size. Method 1 only uses the estimated propagation paths for the ray tracing procedure, method 2 one will first generate new virtual paths by sampling input parameters from a normal distribution utilizing the estimated means and the estimated variances of the propagation paths.

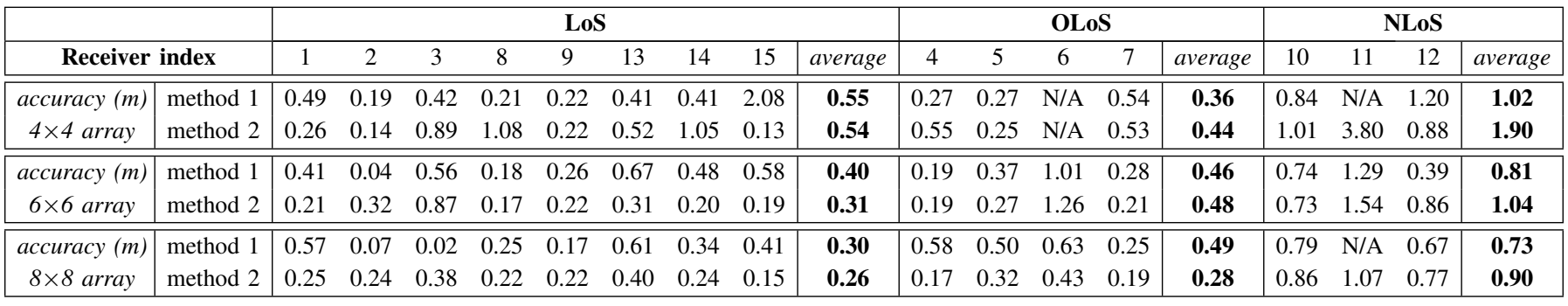

of these paths. We can also see that increasing the antenna array size results in a better localization of the target receiver nodes. Looking at the LoS scenario for method 2, the average accuracy is $0.54 \mathrm{~m}$ with a $4 \times 4$ array, $0.31 \mathrm{~m}$ with a $6 \times 6$ array, and $0.26 \mathrm{~m}$ with a $8 \times 8$ array. This is mainly due to the fact that the physical propagation paths in the environment can be estimated more accurately when increasing the size of the antenna array (resulting in more observations of the radio channel). In an OLoS scenario for method 2 , the average accuracy is $0.44 \mathrm{~m}$ with a $4 \times 4$ array, $0.48 \mathrm{~m}$ with a $6 \times 6$ array, and $0.28 \mathrm{~m}$ with a $8 \times 8$ array. In an NLoS scenario for method 2, the average accuracy is $1.90 \mathrm{~m}$ with a $4 \times 4$ array, $1.04 \mathrm{~m}$ with a $6 \times 6$ array, and $0.90 \mathrm{~m}$ with a $8 \times 8$ array.

Overall, we can state that our new localization algorithm can estimate the location of a target receiver node with an average accuracy of $0.26 \mathrm{~m}$ in a LoS scenario, $0.28 \mathrm{~m}$ in an OLoS scenario, and $0.90 \mathrm{~m}$ in an NLoS scenario.

We now analyze the $8 \times 8$ antenna array configuration more closely, which is supposed to have the best resolution with respect to the estimation accuracy of the propagation paths. When increasing the size of the antenna array configuration, the total number of observations of the radio channel also increases. The measurements with a $M_{T} \times M_{R}=8 \times 8$ antenna array have 4 times as many as observations as the $4 \times 4$ antenna array. Increasing the number of observations causes the estimated variance on the extracted propagation path parameters to decrease. We hypothesize that this is the reason why the $4 \times 4$ antenna array sometimes yields better results, since its larger variance can allow for a broader sampling, which causes large sparse clusters of estimated receiver positions. This is in contrast to the small dense clusters generated by the $8 \times 8$ antenna array.

We found that with the method 1 of only using the estimated mean values of the propagation paths for the localization, an average of 2.5 paths were found in the optimal cluster in a LoS scenario, 2.5 paths in an OLoS scenario, and 2 paths in an NLoS scenario. This corresponds with a usage of $47 \%$ in LoS, $59 \%$ in OLoS and $67 \%$ in NLoS of the total number of paths satisfying the ray matching criterion. By applying our new localization algorithm with the addition of incorporating the estimated variance of the propagation paths, the absolute number of paths in the optimal cluster obviously increased, since we have sampled virtual paths from the original data set of propagation paths. We found that the relative number of paths in the optimal cluster was now
$9 \%$ in LoS, $6 \%$ in OLoS and $14 \%$ in NLoS of the total number of paths satisfying the ray matching criterion. An interesting conclusion is that looking at these relative numbers of paths used, these percentages have dropped significantly compared to method 1 . This indicates that, relatively speaking, more paths were deleted after pruning, and more distinctive clusters of estimated receiver locations could be formed. As an apparent result when looking at the localization accuracy, our proposed method 2 was able to choose the most likely receiver location from several more possible clusters compared to method 1.

Moreover, we can state that in a LoS scenario, the average accuracy of method 1 and method 2 are comparable, regardless of antenna array size. The largest improvements that are made by adding the variance of the propagation paths in our framework, is that it allows for a robuster estimation of the receiver indices. For the $8 \times 8$ antenna array, the average accuracy improves from $0.49 \mathrm{~cm}$ in an OLoS scenario with method 1 to $0.28 \mathrm{~m}$ with method 2. Moreover, we can see that more receiver indices can be estimated by using method 2 over method 1.

\section{CONCLUSiON}

This work presented a novel localization algorithm based on UWB channel sounding, exploiting the geometrical properties of the estimated propagation paths and their corresponding variances. An extension to the high-resolution RiMAX algorithm was developed, after which we tested this framework by performing channel sounding measurements in an indoor laboratory environment. Several multipath parameters were estimated from these measurements, and tracked as a function of frequency. These were subsequently used in a new variancebased localization scheme, allowing for the localization of a target receiver node in LoS-, OLoS- and NLoS scenarios. Our measurements reveal that our technique achieves an average accuracy of $0.26 \mathrm{~m}$ in LoS-, $0.28 \mathrm{~m}$ in OLoS-, and $0.90 \mathrm{~m}$ in NLoS scenarios.

Future research consists of a large measurement campaign with more OLoS and NLoS scenarios, since it was shown that our technique already performs quite well in LoS scenarios. We will also focus on more realistic office environments, since a typical laboratory environment such as the one in this work can be categorized as a very cluttered environment, which can complicate the localization, and deteriorate the true performance of our proposed technique. 


\section{ACKNOWLEDGMENT}

Brecht Hanssens is funded by a Ph.D. grant of the Agency for Innovation by Science and Technology in Flanders (IWT). Emmeric Tanghe is a Post-Doctoral Fellow of the Research Foundation Flanders (FWO). This research was supported by the FWO project G027714N, and by the project IUAP BESTCOM, "BElgian network on STochastic modelling, analysis, design and optimization of COMmunication systems".

This work was carried out in the framework of COST Action CA15104 IRACON.

\section{REFERENCES}

[1] J. Xu, M. Ma, and C. L. Law. AOA Cooperative Position Localization. In IEEE Global Telecommunications Conference (GLOBECOM), pages $1-5,2008$.

[2] S. Kawakami and T. Ohtsuki. Virtual Array Composition for Iterative AOA Localization Sharing Snapshots of Coherent Subarrays. In IEEE International Conference on Wireless Information Technology and Systems (ICWITS), pages 1-4, 2010.

[3] K. C. Cheok, M. Radovnikovich, P. K. Vempaty, G. R. Hudas, J. L. Overholt, and P. Fleck. UWB tracking of mobile robots. In IEEE 21st International Symposium on Personal Indoor and Mobile Radio Communications (PIMRC), pages 2615-2620, 2010.

[4] E. Okamoto, M. Horiba, K. Nakashima, T. Shinohara, and K. Matsumura. Particle swarm optimization-based low-complexity threedimensional UWB localization scheme. In 6th International Conference on Ubiquitous and Future Networks (ICUFN), pages 120-124, 2014.

[5] J. Trogh, D. Plets, A. Thielens, L. Martens, and W. Joseph. Enhanced Indoor Location Tracking Through Body Shadowing Compensation. IEEE Sensors Journal, 16(7):2105-2114, 2016.

[6] J.-R. Jiang, Lin C.-M., F.-Y. Lin, and Huang S.-T. ALRD AoA Localization with RSSI Differences of Directional Antennas for Wireless Sensor Networks. In International Conference on Information Society (i-Society), pages 304-309, 2012.

[7] S. Gezici, Zhi Tian, G.B. Giannakis, Hisashi Kobayashi, A.F. Molisch, H.V. Poor, and Z. Sahinoglu. Localization via ultra-wideband radios: a look at positioning aspects for future sensor networks. IEEE Signal Processing Magazine, 22(4):70-84, 2005.

[8] F. Viani, P. Rocca, G. Oliveri, D. Trinchero, and A. Massa. Localization, tracking, and imaging of targets in wireless sensor networks: An invited review. Radio Science, 46(5):n/a-n/a, 2011. RS5002.

[9] L. Zwirello, T. Schipper, M. Harter, and T. Zwick. Uwb localization system for indoor applications: Concept, realization and analysis. JECE, pages $1-11,2012$.

[10] R. Zetik, E. Malz, G. Shen, H.I. Willms, H. Yan, J. Thielecke, L. Reichardt, M. Janson, R. Salman, R.S. Thomä, et al. Cooperative Localization and Object Recognition in Autonomous UWB Sensor Networks. INTECH Open Access Publisher, 2013.

[11] S. Van de Velde and H. Steendam. CUPID Algorithm for Cooperative Indoor Multipath-Aided Localization. In International Conference on Indoor Positioning and Indoor Navigation (IPIN), pages 1-6, 2012

[12] S. Van de Velde, G. Aurora, L. Vallozzi, H. Rogier, and H. Steendam. Cooperative hybrid localization using Gaussian processes and belief propagation. In IEEE International Conference on Communications Workshops, pages 785-790, 2015.

[13] A. Richter. Estimation of Radio Channel Parameters: Models and Algorithms. PhD thesis, Technische Universität Ilmenau, Fakultät für Elektrotechnik und Informationstechnik, Ilmenau, Germany, 2005.

[14] C. Bin, L. Bin, and P. Zhisong. Robust Location Estimation with Possibilistic Clustering. In ISECS International Colloquium on Computing, Communication, Control, and Management (CCCM), pages 312-315, 2009.

[15] Electro-Metrics. Omni-Directional EM-6865 Wideband Antenna $2 \mathrm{GHz}-18 \mathrm{GHz}$. https://electro-metrics.com/wp-content/uploads/2016/ 12/6865_New_Version-PAS.pdf [Online; accessed 16-May-2017].

[16] P. Laly, D. P. Gaillot, M. Liénard, P. Degauque, E. Tanghe, W. Joseph, and L. Martens. Flexible real-time MIMO channel sounder for multidimensional polarimetric parameter estimation. In IEEE Conference on Antenna Measurements \& Applications (CAMA), page 3. IEEE, 2015.
[17] R. S. Thoma, M. Landmann, G. Sommerkorn, and A. Richter. Multidimensional high-resolution channel sounding in mobile radio. In Proceedings of the 21st IEEE Instrumentation and Measurement Technology Conference (IEEE Cat. No.04CH37510), volume 1, pages 257262, 2004.

[18] J. Poutanen, K. Haneda, J. Salmi, V. M. Kolmonen, A. Richter, P. Almers, and P. Vainikainen. Development of Measurement-Based Ray Tracer for Multi-Link Double Directional Propagation Parameters. In 3rd European Conference on Antennas and Propagation (EUCAP), pages 2622-2626, March 2009.

[19] J. Poutanen, J. Salmi, K. Haneda, V. M. Kolmonen, F. Tufvesson, and P. Vainikainen. Propagation Characteristics of Dense Multipath Components. IEEE Antennas and Wireless Propagation Letters, 9:791794, 2010.

[20] F. Quitin, C. Oestges, F. Horlin, and P. De Doncker. Diffuse Multipath Component Characterization for Indoor MIMO Channels. In European Conference on Antennas and Propagation (EUCAP), pages 1-5, 2010.

[21] B. Hanssens, E. Tanghe, D. P. Gaillot, M. Liénard, C. Oestges, D. Plets, L. Martens, and W. Joseph. An Extension of the RiMAX Multipath Estimation Algorithm for Ultra-Wideband Channel Modeling. EURASIP Journal on Wireless Communications and Networking, submitted in March 2017.

[22] J. A. Fessler and A. O. Hero. Space-Alternating Generalized Expectation-Maximization Algorithm. IEEE Transactions on Signal Processing, 42(10):2664-2677, 1994.

[23] E. Tanghe, D. P. Gaillot, M. Liénard, L. Martens, and W. Joseph. Experimental Analysis of Dense Multipath Components in an Industrial Environment. IEEE Transactions on Antennas and Propagation, 62(7):3797-3805, 2014.

[24] COST 259 Action. http://www.lx.it.pt/cost259 [Online; accessed 18May-2017].

[25] COST 273 Action. http://www.lx.it.pt/cost273 [Online; accessed 18May-2017].

[26] D.-J. Kim, Y.-W. Park, and K.-J. Park. A Novel Validity Index for Determination of the Optimal Number of Clusters. IEICE Transactions on Information and Systems, 84(2):281-285, 2001.

[27] B. Hanssens, D. Plets, E. Tanghe, C. Oestges, D. P. Gaillot, M. Liénard, L. Martens, and W. Joseph. An Indoor Localization Technique Based on Ultra-Wideband AoD/AoA/ToA Estimation. In IEEE International Symposium on Antennas and Propagation/USNC-URSI National Radio Science Meeting, pages 1445-1446, 2016. 